\title{
Quarkonium evolution and suppression in a strongly-coupled quark-gluon plasma
}

\section{Antonio Vairo*}

Physik Department, Technische Universität München, D-85748 Garching, Germany

E-mail: antonio.vairo@tum.de

\begin{abstract}
We compute the quarkonium nuclear modification factor in a strongly coupled quark-gluon plasma for quarkonia that are $S$-wave Coulombic bound states. We perform the analysis in a non-relativistic effective field theory framework that is accurate at leading-order in the heavyquark density expansion and at next-to-leading order in the multipole expansion. We write and solve the Lindblad equation for the heavy quark-antiquark density. Thermal mass shift, width and the Lindblad equation depend on only two non-perturbative parameters: the heavy-quark momentum diffusion coefficient and its dispersive counterpart. Finally, we provide numerical results for the nuclear modification factors of the $1 S$ and $2 S$ bottomonium states.
\end{abstract}

VIII International Workshop On Charm Physics

5-9 September, 2016

Bologna, Italy

${ }^{*}$ Speaker. 


\section{Introduction}

It is thirty years that quarkonium suppression has been suggested as a hard probe for the quark-gluon plasma formed at high energies in heavy-ion collisions [1]. On the experimental side, quarkonium provides a potentially clean signal through dilepton decays [2]. On the theoretical side, since quarkonium is made of heavy quarks, effective field theories and lattice QCD allow to factorize high-energy from low-energy effects and, in principle, compute the suppression from first principles $[3,4,5]$. However several effects enter the final observable: the hydrodynamical evolution of the plasma, production, dissociation and regeneration of quarkonium in the different medium conditions and many others [6].

The problem may be simplified if one considers the quarkonium ground state and particularly the bottomonium $1 S$ states; to a lesser extent the same applies to the charmonium ground state and to the bottomonium $2 S$ states. In this case one may argue that $(a)$ the mass $m$ of the heavy quark is the largest scale of the problem, which qualifies the system as non-relativistic; $(b)$ the inverse Bohr radius, $1 / a_{0}$, is the next relevant scale. For this scale is then larger than $\Lambda_{\mathrm{QCD}}$ and any other thermal scale, it is of order $m \alpha_{\mathrm{s}}$. The hierarchy of energy scales that the system realizes is therefore

$$
m \gg \frac{1}{a_{0}} \sim m \alpha_{\mathrm{s}} \gg \text { any other scale. }
$$

Among the other scales are the thermodynamical scales characterizing the quark-gluon plasma.

If the heavy quark-antiquark system satisfies (1.1), then it is mostly Coulombic. This means that its interaction is described at leading order by a Coulomb potential. One may distinguish between the Coulomb potential of a quark-antiquark pair in a color-singlet configuration, $V_{s}(r)=$ $-C_{F} \alpha_{\mathrm{S}} / r$, and in a color-octet configuration, $V_{o}(r)=\alpha_{\mathrm{s}} /\left(2 N_{c} r\right) . N_{c}=3$ is the number of colors and $C_{F}=\left(N_{c}^{2}-1\right) /\left(2 N_{c}\right)=4 / 3$ is the Casimir of the fundamental representation. In a Coulombic system the typical scale at which the strong coupling is computed in the potential is of the order of $1 / a_{0}$.

Bottomonium suppression has been measured at high energy by the LHC experiments [7, 8, 9]. Theoretically, besides color screening, which was the original suppression mechanism proposed in [1], at least two further mechanisms of quarkonium suppression have been identified over the years: gluodissociation $[10,11]$ and dissociation via parton scattering $[12,13]$. Dissociation mechanisms have been studied in an effective field theory framework applied to Coulombic heavy quarkantiquark states in a weakly-coupled plasma in $[14,15,16,17,18,19]$. An extensive phenomenological analysis of bottomonium suppression can be found in [20, 21, 22].

In a weakly-coupled plasma one assumes the hierarchy $\pi T \gg m_{D}$, where $T$ is the temperature and $m_{D} \sim g T$ the Debye mass of the plasma. The theoretical advantage of this situation is that one may use perturbation theory as a computational tool. It is uncertain, however, if a weakly-coupled plasma is what describes best the medium formed in heavy-ion collisions at the LHC. A more conservative approach consists in assuming that the plasma is strongly coupled, i.e., $\pi T \sim m_{D}$. This is the situation that we will analyze in the following.

We will assume the hierarchy of scales

$$
m \gg \frac{1}{a_{0}} \sim m \alpha_{\mathrm{s}} \gg \pi T \sim m_{D} \gg \text { any other scale, }
$$


where the other scales include the binding energy and $\Lambda_{\mathrm{QCD}}$, whose relative hierarchy is not specified. In the situation (1.2), we will first compute the quarkonium thermal decay width and mass shift in Sec. 2, then we will write and solve the heavy quark-antiquark evolution equations in Sec. 3 and finally compute the bottomonium nuclear modification factor in Sec. 4. This presentation is based on $[23,24]$.

\section{Quarkonium decay width and mass shift in a strongly-coupled plasma}

Under the condition (1.2), the effective field theory suited to describe heavy quark-antiquark pairs at an energy scale lower than $m \alpha_{\mathrm{s}}$ but larger than the thermal scales is potential non-relativistic QCD (pNRQCD) [25, 26, 27]. According to our hierarchy of energy scales, pNRQCD may be computed setting to zero the temperature and any other scale lower than the inverse Bohr radius. The remaining scales provide non-perturbative contributions: contributions from thermal scales have to be resummed to all orders because they are induced by a strongly-coupled plasma, but also contributions scaling with the binding energy of the system may be non-perturbative if the binding energy is of the order of or smaller than $\Lambda_{\mathrm{QCD}}$. The Lagrangian of pNRQCD at next-to-leading order in the multipole expansion reads

$$
\begin{aligned}
\mathscr{L}_{\mathrm{pNRQCD}}= & \int d^{3} r \operatorname{Tr}\left[S^{\dagger}\left(i \partial_{0}-h_{s}\right) S+O^{\dagger}\left(i D_{0}-h_{o}\right) O\right] \\
& +\operatorname{Tr}\left[O^{\dagger} \mathbf{r} \cdot g \mathbf{E} S+S^{\dagger} \mathbf{r} \cdot g \mathbf{E} O+\frac{1}{2}\left(O^{\dagger} \mathbf{r} \cdot g \mathbf{E} O+O^{\dagger} O \mathbf{r} \cdot g \mathbf{E}\right)\right] \\
& +\mathscr{L}_{\text {light }},
\end{aligned}
$$

where $r$ is the distance between the heavy quark and the antiquark (the above Lagrangian is accurate up to order $r$ ), $S$ and $O=\sqrt{2} O^{a} T^{a}$ stand for the heavy quark-antiquark fields in a color-singlet and a color-octet configuration respectively, $h_{s}=\mathbf{p}^{2} / m+V_{s}$ is the color-singlet Hamiltonian, $h_{o}=$ $\mathbf{p}^{2} / m+V_{o}$ is the color-octet Hamiltonian, $\mathbf{E}$ is the chromoelectric field and $g$ the strong coupling. The term $\mathscr{L}_{\text {light }}$ stands for the QCD Lagrangian with light quarks only. The covariant derivative acting on the octet field $O$ in (2.1) can be eliminated by means of suitable field redefinitions: $O \rightarrow \Omega O \Omega^{\dagger}$ and $\mathbf{E} \rightarrow \Omega \mathbf{E} \Omega^{\dagger}$, where $\Omega=\mathrm{P} \exp \left[-i g \int_{-\infty}^{t} d s A_{0}(s, \mathbf{R})\right]$ and $\mathbf{R}$ is the center of mass coordinate; P stands for path ordering. We make implicitly use of these field redefinitions in Sec. 3.

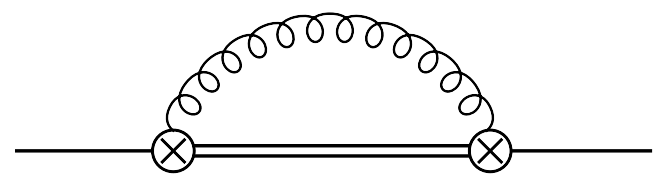

Figure 1: The leading color-singlet self-energy diagram in pNRQCD. The single line is a singlet heavy quark-antiquark propagator, the double line an octet heavy quark-antiquark propagator, the curly line stands for gluons and the vertices are chromoelectric dipole vertices.

At order $r$ in the multipole expansion quark-antiquark pairs interact with the medium through chromoelectric-dipole interactions. In (2.1) we have set equal to one the matching coefficients of 
these terms, since higher-order corrections are beyond our accuracy. The color-singlet self energy shown in Fig. 1 reads

$$
\Sigma_{s}(t)=\frac{g^{2}}{2 N_{c}} \int_{t_{0}}^{t} d t_{2} r^{i} e^{-i h_{o}\left(t-t_{2}\right)} r^{j} e^{i h_{s}\left(t-t_{2}\right)}\left\langle E^{a, i}(t, \mathbf{0}) E^{a, j}\left(t_{2}, \mathbf{0}\right)\right\rangle,
$$

where $\langle\cdots\rangle$ stands for the thermal average. The time $t_{0}$ is the formation time of the quark-gluon plasma, i.e., the initial time for the evolution of the heavy quark-antiquark pairs in the medium. In the following, we will assume that $t-t_{0}$ is larger than any other time scale of the system, so that we can approximate $\int_{t_{0}}^{t} d t_{2} f\left(t_{2}\right) \approx \int_{0}^{\infty} d s f(t-s)$, and that the evolution of the temperature is quasistatic, $1 / T \times d T / d t \sim 1 / t \lesssim$ binding energy, so that we may indeed compute the thermal correlator of two chromoelectric fields as if the medium was in thermal equilibrium at a slowly varying temperature $T$.

The leading contribution to the thermal decay width is provided by the imaginary part of the color-singlet self energy. Since we assume that the thermodynamical scales are much larger than the binding energy, we may set it equal to zero. This greatly simplifies the final expression, which reads for $1 S$ states

$$
\Gamma=-2\left\langle\operatorname{Im}\left(-i \Sigma_{s}\right)\right\rangle=3 a_{0}^{2} \kappa .
$$

The heavy-quark momentum diffusion coefficient, $\kappa$, is defined as [28, 29]

$$
\kappa=\frac{g^{2}}{6 N_{c}} \operatorname{Re} \int_{-\infty}^{+\infty} d s\left\langle\mathrm{~T} E^{a, i}(s, \mathbf{0}) E^{a, i}(0, \mathbf{0})\right\rangle=\frac{g^{2}}{6 N_{c}} \int_{0}^{\infty} d s\left\langle\left\{E^{a, i}(s, \mathbf{0}), E^{a, i}(0, \mathbf{0})\right\}\right\rangle,
$$

where $\mathrm{T}$ stands for time ordering. A recent lattice determination found for $\kappa$ [30]:

$$
1.8 \lesssim \frac{\kappa}{T^{3}} \lesssim 3.4
$$

This estimate has been obtained from a pure $\mathrm{SU}(3)$ plasma at a temperature of about $1.5 T_{c}$. With this value of $\kappa, m_{b}=4.8 \mathrm{GeV}$ and $1 / a_{0}=1.334 \mathrm{GeV}$ that follows from the self-consistency equation $1 / a_{0}=m_{b} C_{F} \alpha_{\mathrm{s}}\left(1 / a_{0}\right) / 2$, we obtain for the $\Upsilon(1 S): 3.0 \mathrm{GeV}^{-2} T^{3} \lesssim \Gamma_{\Upsilon(1 S)} \lesssim 5.7 \mathrm{GeV}^{-2} T^{3}$. This is a rather large thermal width for temperatures close or above the cross-over temperature to the quark-gluon plasma, $T_{c}$, which is about $150 \mathrm{MeV}[31,32,33]$.

The leading contribution to the quarkonium thermal mass shift is provided by the real part of the color-singlet self energy. Setting again to zero terms of the order of the binding energy gives for $1 S$ states

where

$$
\delta m=\left\langle\operatorname{Re}\left(-i \Sigma_{s}\right)\right\rangle=\frac{3}{2} a_{0}^{2} \gamma
$$

$$
\gamma=\frac{g^{2}}{6 N_{c}} \operatorname{Im} \int_{-\infty}^{+\infty} d s\left\langle\mathrm{~T} E^{a, i}(s, \mathbf{0}) E^{a, i}(0, \mathbf{0})\right\rangle=-i \frac{g^{2}}{6 N_{c}} \int_{0}^{\infty} d s\left\langle\left[E^{a, i}(s, \mathbf{0}), E^{a, i}(0, \mathbf{0})\right]\right\rangle .
$$

So far $\gamma$ has not been computed on the lattice. The only estimate we have for $\gamma$ is the perturbative calculation done at leading order in [14]:

$$
\gamma=-2 \zeta(3) C_{F}\left(\frac{4}{3} N_{c}+n_{f}\right) \alpha_{\mathrm{s}}^{2} T^{3}
$$

where $\zeta$ is the Riemann zeta function and $n_{f}=4$ is the number of active flavours at the bottommass scale. We should, however, consider that perturbation theory neither seems to converge in the case of $\kappa$ [34] nor agrees at leading order with the lattice result [35]. 


\section{Evolution equations in a strongly-coupled plasma}

The yield of quarkonium $n S$ states in ion-ion collisions normalized with respect to the yield in $p p$ collisions, as measured from dilepton decays, is called the quarkonium nuclear modification factor, $R_{A A}(n S)$. It can be expressed as the density of (color singlet) $n S$ states in ion-ion collisions normalized with respect to the same quantity in $p p$ collisions $[2,24]$. The density of color-singlet heavy quark-antiquark states, $\rho_{s}$, and color-octet ones, $\rho_{o}^{b a}=\rho_{o} \delta^{a b} /\left(N_{c}^{2}-1\right)$, may be expressed in the close-time-path formalism as a singlet and octet propagator respectively that propagate from the upper branch (labeled 1) to the lower branch (labeled 2) of the time path [36]

$$
\begin{aligned}
\left\langle\mathbf{r}^{\prime}\left|\rho_{s}(t ; t)\right| \mathbf{r}\right\rangle & =\operatorname{Tr}\left\{\rho S^{\dagger}(t, \mathbf{r}, \mathbf{R}) S\left(t, \mathbf{r}^{\prime}, \mathbf{R}\right)\right\}=\left\langle S_{1}\left(t, \mathbf{r}^{\prime}, \mathbf{R}\right) S_{2}^{\dagger}(t, \mathbf{r}, \mathbf{R})\right\rangle, \\
\frac{\delta^{a b}}{N_{c}^{2}-1}\left\langle\mathbf{r}^{\prime}\left|\rho_{o}(t ; t)\right| \mathbf{r}\right\rangle & =\operatorname{Tr}\left\{\rho O^{a \dagger}(t, \mathbf{r}, \mathbf{R}) O^{b}\left(t, \mathbf{r}^{\prime}, \mathbf{R}\right)\right\}=\left\langle O_{1}^{b}\left(t, \mathbf{r}^{\prime}, \mathbf{R}\right) O_{2}^{a \dagger}(t, \mathbf{r}, \mathbf{R})\right\rangle .
\end{aligned}
$$

We have assumed that the heavy quarks comove with the medium, so that we do not need to consider the center of mass motion.

The evolution equations for the singlet and octet densities may be computed, therefore, by considering diagrams similar to the one shown in Fig. 1, but now describing the evolution of the $\left\langle S_{1} S_{2}^{\dagger}\right\rangle$ and $\left\langle O_{1}^{b} O_{2}^{a \dagger}\right\rangle$ propagators. Keeping only terms linear in the heavy-quark densities and resumming a certain class of self-energy contributions by means of a Schwinger-Dyson equation, we obtain the evolution equations

$$
\begin{aligned}
& \frac{d \rho_{s}(t ; t)}{d t}=-i\left[h_{s}, \rho_{s}(t ; t)\right]-\Sigma_{s}(t) \rho_{s}(t ; t)-\rho_{s}(t ; t) \Sigma_{s}^{\dagger}(t)+\Xi_{s o}\left(\rho_{o}, t\right), \\
& \frac{d \rho_{o}(t ; t)}{d t}=-i\left[h_{o}, \rho_{o}(t ; t)\right]-\Sigma_{o}(t) \rho_{o}(t ; t)-\rho_{o}(t ; t) \Sigma_{o}^{\dagger}(t)+\Xi_{o s}\left(\rho_{s}, t\right)+\Xi_{o o}\left(\rho_{o}, t\right) .
\end{aligned}
$$

The explicit expressions of the functions $\Sigma_{o}, \Xi_{s o}, \Xi_{o s}$ and $\Xi_{o o}$ are similar to (2.2), i.e., they are time integrals that depend on the energies of the states and on the chromoelectric field correlator [24]. The function $\Sigma_{o}$ is the color-octet self energy; differently from the color-singlet self energy it gets the contributions of two diagrams since chromoelectric fields allow for both octet-singlet and octet-octet transitions. The functions $\Xi_{s o}, \Xi_{o s}$ and $\Xi_{o o}$ depend also on the densities of heavy quarkantiquark states, hence, equations (3.3) and (3.4) show the coupled evolution of the singlet and octet densities. Their interpretation is straightforward: the function $\Xi_{s o}$ accounts for the production (or regeneration) of singlets through the decay of octets, while the functions $\Xi_{o s}$ and $\Xi_{o o}$ account for the production of octets through the decays of singlets and octets respectively. Also these two octet production mechanisms can be traced back to the two different sets of chromoelectric-dipole operators in the pNRQCD Lagrangian (2.1).

The evolution equations simplify considerably for the hierarchy of energy scales (1.2). In particular, if $T$ is larger than the binding energy we may set to one the energy-dependent exponentials. 
This allows to write

$$
\begin{aligned}
\Sigma_{s}(t) & =\frac{r^{2}}{2}[\kappa(t)+i \gamma(t)], \\
\Sigma_{o}(t) & =\frac{N_{c}^{2}-2}{2\left(N_{c}^{2}-1\right)} \frac{r^{2}}{2}[\kappa(t)+i \gamma(t)], \\
\Xi_{s o}\left(\rho_{o}, t\right) & =\frac{1}{N_{c}^{2}-1} r^{i} \rho_{o} r^{i} \kappa(t), \\
\Xi_{o s}\left(\rho_{s}, t\right) & =r^{i} \rho_{s} r^{i} \kappa(t), \\
\Xi_{o o}\left(\rho_{o}, t\right) & =\frac{N_{c}^{2}-4}{2\left(N_{c}^{2}-1\right)} r^{i} \rho_{o} r^{i} \kappa(t),
\end{aligned}
$$

where $\kappa$ is the heavy-quark momentum diffusion coefficient defined in (2.4) and $\gamma$ has been defined in (2.7). They depend on time through the temperature.

With the above functions, we can rewrite the evolution equations (3.3) and (3.4) in the Lindblad form [37, 38]:

$$
\frac{d \rho}{d t}=-i[H, \rho]+\sum_{n}\left(C_{n} \rho C_{n}^{\dagger}-\frac{1}{2}\left\{C_{n}^{\dagger} C_{n}, \rho\right\}\right)
$$

where $\rho$ is the matrix

$$
\rho=\left(\begin{array}{cc}
\rho_{s} & 0 \\
0 & \rho_{o}
\end{array}\right)
$$

$H$ is the Hermitian operator

$$
H=\left(\begin{array}{cc}
h_{s} & 0 \\
0 & h_{o}
\end{array}\right)+\frac{r^{2}}{2} \gamma(t)\left(\begin{array}{cc}
1 & 0 \\
0 & \frac{N_{c}^{2}-2}{2\left(N_{c}^{2}-1\right)}
\end{array}\right),
$$

and the operators $C_{n}$ are the six collapse operators $C_{i}^{0}$ and $C_{i}^{1}$ given by

$$
\begin{aligned}
C_{i}^{0} & =\sqrt{\frac{\kappa(t)}{N_{c}^{2}-1}} r^{i}\left(\begin{array}{cc}
0 & 1 \\
\sqrt{N_{c}^{2}-1} & 0
\end{array}\right), \\
C_{i}^{1} & =\sqrt{\frac{\left(N_{c}^{2}-4\right) \kappa(t)}{2\left(N_{c}^{2}-1\right)}} r^{i}\left(\begin{array}{ll}
0 & 0 \\
0 & 1
\end{array}\right) .
\end{aligned}
$$

The Lindblad equation has been studied in relation with quarkonium in a quark-gluon plasma also in [39].

\section{Bottomonium suppression}

We assume that the temperature of the quark-gluon plasma evolves slowly according to [40]

$$
T=T_{0}\left(\frac{t_{0}}{t}\right)^{v_{s}^{2}}
$$

where $T_{0}$ is the initial temperature and $v_{s}$ is the velocity of sound in the medium. In a deconfined plasma at a very high temperature $v_{s}^{2}=1 / 3$. As values of $T_{0}$ and $t_{0}$ for central collisions at the LHC we take $T_{0}=475 \mathrm{MeV}$ and $t_{0}=0.6 \mathrm{fm} \mathrm{[41].}$ 
We study collisions with different centralities. Since we assume the plasma to be homogeneous and isotropic, the only effect that a difference in centrality produces is a change in the initial value of the energy density and hence in $T_{0}$. Values of $T_{0}$ for different centralities and mean impact parameters are listed in Tab. 1.

\begin{tabular}{|c|c|c|}
\hline centrality $(\%)$ & $\langle b\rangle(\mathrm{fm})$ & $T_{0}(\mathrm{MeV})$ \\
\hline $0-10$ & 3.4 & 471 \\
$10-20$ & 6.0 & 461 \\
$20-30$ & 7.8 & 449 \\
$30-50$ & 9.9 & 425 \\
$50-100$ & 13.6 & 304 \\
\hline
\end{tabular}

Table 1: Initial temperature of the fireball, $T_{0}$, for different centrality bins and mean impact parameters, $\langle b\rangle$ [42]; 0-10\% centrality means that from all the collisions we select the most central $10 \%$.

According to (4.1) and Tab. 1, if the evolution starts at $t_{0}=0.6 \mathrm{fm}$, the fireball has cooled down to about $250 \mathrm{MeV}$ at about $4 \mathrm{fm}$ for the most central collisions and at about $1.1 \mathrm{fm}$ for the most peripheral ones. A temperature of about $250 \mathrm{MeV}$ is the smallest temperature, still larger than the cross-over temperature to the quark-gluon plasma, $T_{c}$, where we expect the hierarchy (1.2) to be safely realized for the bottomonium lowest states.

\begin{tabular}{|c|c|c|c|}
\hline \multicolumn{2}{|c|}{$30-50 \%$ centrality } & \multicolumn{2}{c|}{$50-100 \%$ centrality } \\
\hline$R_{A A}(1 S)$ & $\frac{R_{A A}(2 S)}{R_{A A}(1 S)}$ & $R_{A A}(1 S)$ & $\frac{R_{A A}(2 S)}{R_{A A}(1 S)}$ \\
\hline $0.23_{-0.07}^{+0.10}$ & $0.24 \pm 0.09$ & $0.80 \pm 0.05$ & $0.59 \pm 0.10$ \\
\hline
\end{tabular}

Table 2: Results for $R_{A A}(1 S)$ and $R_{A A}(2 S)$ for $\kappa / T^{3}$ in the lattice range, $\gamma=0$ and $\delta=1$ in the bottomonium case.

In Tab. 2 we show the bottomonium nuclear modification factors for $\kappa$ in the lattice range (2.5), $\gamma=0, \delta=1$ and for centralities $30-50 \%$ and $50-100 \%$. The number $\delta / \alpha_{\mathrm{s}}\left(m_{b}\right)$ provides the initial fraction of octets with respect to singlets. We correct for feed-down effects using the method of [22]. A scan of the nuclear modification factor for different values of $\gamma$ and $\delta$ shows that the results are sensitive to $\gamma$ and seem to prefer a small value, whereas they are rather insensitive to $\delta$.

The results can be compared with the experimental observations of [7]. The CMS collaboration measures for 30-40\% centrality, $R_{A A}(1 S)=0.681 \pm 0.069 \pm 0.085 \pm 0.093$ and $R_{A A}(2 S) /$ $R_{A A}(1 S)=0.344 \pm 0.138 \pm 0.027 \pm 0.041$, for $40-50 \%$ centrality, $R_{A A}(1 S)=0.590 \pm 0.096 \pm$ $0.086 \pm 0.08$ and $R_{A A}(2 S) / R_{A A}(1 S)=0.420 \pm 0.220 \pm 0.048 \pm 0.050$, and finally for $50-100 \%$ centrality, $R_{A A}(1 S)=1.005 \pm 0.121 \pm 0.176 \pm 0.137$ and $R_{A A}(2 S) / R_{A A}(1 S)=0.304 \pm 0.154 \pm 0.040 \pm$ 0.037 .

\section{Conclusions}

In this work, we have considered quarkonium and more specifically bottomonium suppression in a strongly-coupled plasma. Relatively little is known from first principles about this case, al- 
though it is a case of particular interest at the LHC, for the temperature may not be much larger than $m_{D}$.

We have computed the leading thermal decay width (2.3) and mass shift (2.6) induced by the plasma on $1 S$-state quarkonia. They depend on only two non-perturbative parameters, respectively the heavy-quark momentum diffusion coefficient $\kappa$ and the parameter $\gamma$.

Interestingly, for a strongly-coupled plasma satisfying the hierarchy (1.2) the leading-order evolution equations for the singlet and octet densities reduce to a Lindblad equation that depends on the same two parameters $\kappa$ and $\gamma$. We have computed the time evolution of the heavy quarkantiquark densities and the nuclear modification factors $R_{A A}(1 S)$ and $R_{A A}(2 S)$ for bottomonium (see Tab. 2) in the framework of Bjorken's evolution equation of the plasma.

The output depends crucially on the initial conditions and on the parameters $\kappa$ and $\gamma$. While $\kappa$ has at least been computed in quenched lattice QCD for temperatures close to $T_{c}, \gamma$ has not. The determination of $\gamma$ remains therefore one of the main uncertainties in the determination of the modification factor $R_{A A}$.

\section{Acknowledgements}

I wish to thank Nora Brambilla, Miguel Escobedo and Joan Soto for collaboration on the work presented here. This work was supported by the Bundesministerium für Bildung und Forschung (BMBF) through the "Verbundprojekt 05P2015 - ALICE at High Rate (BMBF-FSP 202) GEMTPC Upgrade and Field theory based investigations of ALICE physics" under grant 05P15WOCA1 and by the DFG cluster of excellence "Origin and structure of the universe" (www.universecluster.de).

\section{References}

[1] T. Matsui and H. Satz, Phys. Lett. B 178 (1986) 416.

[2] L. D. McLerran and T. Toimela, Phys. Rev. D 31 (1985) 545.

[3] N. Brambilla et al., Heavy quarkonium physics, CERN-2005-005, (CERN, Geneva, 2005) [arXiv:hep-ph/0412158].

[4] N. Brambilla et al., Eur. Phys. J. C 71 (2011) 1534 [arXiv:1010.5827 [hep-ph]].

[5] N. Brambilla et al., Eur. Phys. J. C 74 (2014) 2981 [arXiv:1404.3723 [hep-ph]].

[6] G. Aarts et al., arXiv:1612.08032 [nucl-th].

[7] S. Chatrchyan et al. [CMS Collaboration], Phys. Rev. Lett. 109 (2012) 222301 [arXiv:1208.2826 [nucl-ex]].

[8] V. Khachatryan et al. [CMS Collaboration], [arXiv:1611.01510 [nucl-ex]].

[9] G. G. Fronzé [ALICE Collaboration], arXiv:1612.06691 [hep-ex].

[10] D. Kharzeev and H. Satz, Phys. Lett. B 334 (1994) 155 [hep-ph/9405414].

[11] X. M. Xu, D. Kharzeev, H. Satz and X. N. Wang, Phys. Rev. C 53 (1996) 3051 [hep-ph/9511331].

[12] L. Grandchamp and R. Rapp, Phys. Lett. B 523 (2001) 60 [hep-ph/0103124]. 
[13] L. Grandchamp and R. Rapp, Nucl. Phys. A 709 (2002) 415 [hep-ph/0205305].

[14] N. Brambilla, J. Ghiglieri, A. Vairo and P. Petreczky, Phys. Rev. D 78 (2008) 014017 [arXiv:0804.0993 [hep-ph]].

[15] N. Brambilla, M. A. Escobedo, J. Ghiglieri, J. Soto and A. Vairo, JHEP 1009 (2010) 038 [arXiv:1007.4156 [hep-ph]].

[16] A. Vairo, AIP Conf. Proc. 1317 (2011) 241 [arXiv:1009.6137 [hep-ph]].

[17] N. Brambilla, M. A. Escobedo, J. Ghiglieri and A. Vairo, JHEP 1112 (2011) 116 [arXiv:1109.5826 [hep-ph]].

[18] N. Brambilla, M. A. Escobedo, J. Ghiglieri and A. Vairo, JHEP 1305 (2013) 130 [arXiv:1303.6097 [hep-ph]].

[19] M. A. Escobedo, F. Giannuzzi, M. Mannarelli and J. Soto, Phys. Rev. D 87 (2013) 114005 [arXiv:1304.4087 [hep-ph]].

[20] B. Krouppa and M. Strickland, Universe 2 (2016) 16 [arXiv:1605.03561 [hep-ph]].

[21] B. Krouppa, R. Ryblewski and M. Strickland, Phys. Rev. C 92 (2015) 061901 [arXiv:1507.03951 [hep-ph]].

[22] M. Strickland and D. Bazow, Nucl. Phys. A 879 (2012) 25 [arXiv:1112.2761 [nucl-th]].

[23] N. Brambilla, M. A. Escobedo, J. Soto and A. Vairo, arXiv:1612.07248 [hep-ph].

[24] N. Brambilla, M. A. Escobedo, J. Soto and A. Vairo, TUM-EFT 89/16, in preparation.

[25] A. Pineda and J. Soto, Nucl. Phys. Proc. Suppl. 64 (1998) 428 [hep-ph/9707481].

[26] N. Brambilla, A. Pineda, J. Soto and A. Vairo, Nucl. Phys. B 566 (2000) 275 [hep-ph/9907240].

[27] N. Brambilla, A. Pineda, J. Soto and A. Vairo, Rev. Mod. Phys. 77 (2005) 1423 [hep-ph/0410047].

[28] J. Casalderrey-Solana and D. Teaney, Phys. Rev. D 74 (2006) 085012 [hep-ph/0605199].

[29] S. Caron-Huot and G. D. Moore, Phys. Rev. Lett. 100 (2008) 052301 [arXiv:0708.4232 [hep-ph]].

[30] A. Francis, O. Kaczmarek, M. Laine, T. Neuhaus and H. Ohno, Phys. Rev. D 92 (2015) 116003 [arXiv:1508.04543 [hep-lat]].

[31] S. Borsanyi et al. [Wuppertal-Budapest Collaboration], JHEP 1009 (2010) 073 [arXiv:1005.3508 [hep-lat]].

[32] A. Bazavov et al. [HotQCD Collaboration], Phys. Rev. D 90 (2014) 094503 [arXiv:1407.6387 [hep-lat]].

[33] A. Bazavov, N. Brambilla, H.-T. Ding, P. Petreczky, H.-P. Schadler, A. Vairo and J. H. Weber, Phys. Rev. D 93 (2016) 114502 [arXiv:1603.06637 [hep-lat]].

[34] S. Caron-Huot and G. D. Moore, JHEP 0802 (2008) 081 [arXiv:0801.2173 [hep-ph]].

[35] D. Banerjee, S. Datta, R. Gavai and P. Majumdar, Phys. Rev. D 85, 014510 (2012) [arXiv:1109.5738 [hep-lat]].

[36] J. Berges, AIP Conf. Proc. 739 (2005) 3 [hep-ph/0409233].

[37] G. Lindblad, Commun. Math. Phys. 48 (1976) 119.

[38] V. Gorini, A. Kossakowski and E. C. G. Sudarshan, J. Math. Phys. 17 (1976) 821. 
[39] Y. Akamatsu, Phys. Rev. D 91 (2015) 056002 [arXiv:1403.5783 [hep-ph]].

[40] J. D. Bjorken, Phys. Rev. D 27 (1983) 140.

[41] W. M. Alberico, A. Beraudo, A. De Pace, A. Molinari, M. Monteno, M. Nardi, F. Prino and M. Sitta, Eur. Phys. J. C 73 (2013) 2481 [arXiv:1305.7421 [hep-ph]].

[42] S. Chatrchyan et al. [CMS Collaboration], Phys. Rev. C 84 (2011) 024906 [arXiv:1102.1957 [nucl-ex]]. 